\section{Deferiprone as adjunctive treatment for patients with invasive mucormycosis: A retrospective case series}

\author{
Maria N. Chitasombat, ${ }^{1}$ \\ Pimjai Niparuck ${ }^{2}$ \\ ${ }^{1}$ Division of Infectious Diseases; and \\ ${ }^{2}$ Division of Hematology, Department of \\ Medicine, Faculty of Medicine \\ Ramathibodi Hospital, Mahidol \\ University, Bangkok, Thailand
}

\begin{abstract}
Mucormycosis is a life-threatening disease requiring multimodal treatment with antifungals and surgery. The mortality rate remains high, prompting consideration of alternative treatment strategies. Deferiprone has in vitro activity against Mucorales, but its efficacy has never been evaluated in humans. Here, we retrospectively analyzed patients with confirmed mucormycosis who received deferiprone from 2011 to 2017. Five patients had hematologic malignancies and one was diabetic. The sites of infection included sinus-orbit-cerebral (67\%), lung (17\%), and disseminated infection (17\%). Surgery was performed in $83 \%$ of cases and achieved local control for $33 \%$ of patients. A combination regimen of polyenes plus echinocandins was administered with stepdown treatment using posaconazole. The median duration of antifungal treatment was 86 days (range: 46-435 days) days. Deferiprone was given as adjunctive treatment with a median dose and duration of $100 \mathrm{mg} / \mathrm{kd} /$ day (range: 86.2-100 $\mathrm{mg} / \mathrm{kg} /$ day) and 25 days (range: 15-215 days), respectively. Overall, deferiprone was well-tolerated. Successful outcomes were observed at 12-week follow-up for $67 \%$ of patients. The mortality rate at 180 day follow-up was $50 \%$. Adjunctive therapy with deferiprone showed safety and tolerability.
\end{abstract}

\section{Introduction}

Mucormycosis is a rare life-threatening mold infection. ${ }^{1}$ Risk factors for invasive mucormycosis (IM) include neutropenia, active hematologic malignancy (especially relapsed leukemia), treatment with highdose corticosteroids, diabetes mellitus (DM) and/or ketoacidosis, transfusion-associated iron overload, and transplantation. ${ }^{1}$
Delays in effective treatment result in increased morbidity and mortality due to the aggressive nature of the infection. ${ }^{2}$ The burden of disease and the cost of treatment is high. ${ }^{3}$ Effective antifungal treatments include amphotericin B-based regimens (lipid formulations of amphotericin B, L$\mathrm{AmB}$ or the less-effective amphotericin B deoxycholate, D-AmB), posaconazole, and isavuconazole. ${ }^{4}$ Surgery has a role in both diagnosis and therapy to allow better penetration of antifungals to the site of infection. ${ }^{4}$ Surgical debridement is recommended in order to cure localized disease and increase survival rates. ${ }^{1}$ Reversal of the underlying predisposing factors such as neutropenia, hyperglycemia and acidosis is important for clinical outcome. ${ }^{1}$ Treatment outcomes remain unsatisfactory even for patients treated with antifungals and surgery. ${ }^{1}$ Most infections occur among patient with hematologic malignancies during neutropenia or relapsed disease. ${ }^{1}$ Transfusionassociated iron overload is frequent among patients with leukemia. ${ }^{5}$ Iron overload plays an important role in the growth and virulence of Mucorales. ${ }^{6}$ Iron chelators have complex interactions with Mucorales: for instance, deferoxamine serves as a siderophore, delivering free iron to support growth of Mucorales, which explains the increased incidence of mucormycosis in patients treated with this drug. ${ }^{6}$ By contrast, deferiprone (DEF) or deferasirox do not supply free iron for the fungus and have fungicidal activity against Mucorales. ${ }^{6}$ Iron deprivation using either deferasirox or DEF showed promising results in an animal model of IM. $^{7-9}$ Specifically, DEF (100 $\mathrm{mg} / \mathrm{kg}$ every other day) showed efficacy equivalent to L-AmB in improving survival of diabetic ketoacidotic mice compared with placebo. ${ }^{7}$ Successful outcomes of deferasirox treatment were first reported in a diabetic patient with refractory rhinocerebral mucormycosis: after failing an 8-month course of L-AmB; a 7-day course of deferasirox significantly improved cerebellar disease. ${ }^{10}$ Data from an open-label study of deferasirox used as adjunctive treatment $(n=8)$ with a median duration of 14 days (range: 7-21 days) showed a promising safety profile and favorable outcomes among diabetic patients and transplant recipients; however, none of these patients were neutropenic. ${ }^{11}$ The results of a randomized controlled trial of deferasirox used as adjunctive therapy for IM (DEFEAT trial) $(n=11)$ revealed higher mortality in deferasirox-treated patients compared with placebo $(n=9) .{ }^{12}$ There are several plausible explanations for this result. Firstly, the preclinical data for deferasirox were generated in diabetic ketoacidosis models of dissemi-
Correspondence: Maria Nina Chitasombat, Assistant Professor, Division of Infectious Disease, Department of Medicine, Faculty of Medicine, Ramathibodi Hospital, Mahidol University, 270 Rama VI Road, Ratchathewi, Bangkok 10400, Thailand.

Tel.: +66.8.1929 9010 - Fax: +66.201.1581.

E-mail: mchitasombat@gmail.com

Key words: Deferiprone; iron chelator; mucormycosis; Mucorales.

Acknowledgements: we thank the patients and medical personnel involved in the study. We thank Edanz Group (www.edanzediting. com/ac) for editing a draft of this manuscript.

Contributions: MNC conceptualized and designed the study, data analysis, drafted and edited the manuscript. PN responsible for edited the manuscript. All authors have given their approval for this version to be published.

Conflict of interest: the authors declare no potential conflicts of interest.

Received for publication: 31 May 2018.

Revision received: 12 July 2018.

Accepted for publication: 6 August 2018.

This work is licensed under a Creative Commons Attribution-NonCommercial 4.0 International License (CC BY-NC 4.0).

CCopyright M.N. Chitasombat and P. Niparuck, 2018

Licensee PAGEPress, Italy

Infectious Disease Reports 2018; 10:7765

doi:10.4081/idr.2018.7765

nated mucormycosis, and the efficacy of iron chelation is less well established in preclinical compared with neutropenic models of mucormycosis. Secondly, the duration of treatment was short, with only one third of patients completing 14 days of therapy. In our opinion, a 14-day course of deferasirox may be too short to demonstrate efficacy and contribute to favorable clinical outcomes in IM. Thirdly, a majority of patients with pulmonary infection were randomized to the deferasirox arm; this condition is associated with higher mortality than rhinocerebral mucormycosis. ${ }^{2,12-16}$ By contrast, an open-label study from India $(n=7)$ evaluated the use of adjunctive deferasirox in patients with IM (mainly rhinocerebral mucormycosis) in whom adequate surgical debridement was not feasible; all patients survived and tolerated the medication well. ${ }^{17}$ However, the individuals studied did not include patients with hematologic malignancies, transplant recipients, neutropenic patients, or patients with pulmonary disease. ${ }^{17}$ Since these studies, none 
of the iron chelators have been evaluated in clinical studies; given the rarity of IM, another randomized control trial is likely to be difficult to conduct. ${ }^{18}$ At our center, treatment options at the time of the study were limited since new antifungals such as intravenous posaconazole or isavuconazole were not available. DEF had not previously been evaluated for treatment of IM in humans. We postulate that adjunctive DEF treatment may be beneficial for the treatment of IM based on preclinical data. The treatment effect of individual iron chelators may be different. Long-term use of DEF was welltolerated in thalassemic patients. ${ }^{19} \mathrm{DEF}$ was approved for the treatment of iron overload in Asia, Europe and United States. ${ }^{20}$ Side effects included transient liver dysfunction, gastrointestinal discomfort, and arthralgia, although none of the patients discontinued therapy..$^{19}$ Long-term use of high-dose DEF (100 mg/kg/day for 2 years) showed higher efficacy in reduction of iron overload without increased toxicity. ${ }^{19}$ In this study, we retrospectively evaluated the safety and tolerability of DEF used as adjunctive treatment for IM.

\section{Materials and Methods}

We retrospectively reviewed medical records of patients with IM who received DEF between January 2011 and December 2017 at our center. All patients had pathology-confirmed IM in accordance with EORTC/MSG criteria. ${ }^{21}$ All patients who received DEF for more than 7 days were included in the study. Patient demographics, underlying diseases, clinical manifestations, and laboratory data were collected. Treatment data, including type of surgery, type and duration of antifungal treatment, and clinical outcome were collected. Treatment response was evaluated at 12-week follow-up using EORTC/MSG criteria, as well as at the end of the study period. ${ }^{22}$ The reasons for DEF discontinuation were collected. Assessment of potential adverse effects during DEF treatment was based on the cancer therapy evaluation program using common terminology criteria for adverse events (CTCAE) version 5.0. Adverse events were graded from 0 to 4 (e.g., grade 2 for aspartate aminotransferase (AST, U/L) and alanine aminotransferase (ALT, U/L) levels >3.0$5.0 \times$ upper limit of normal (ULN); and for alkaline phosphatase (ALP, U/L) and gamma glutamyltransferase (GGT, U/1) levels $>2.5-5.0 \times$ ULN).

Neutropenia was defined as white blood cell counts $<1000 \quad$ cells $/ \mathrm{mm}^{3}$. Thrombocytopenia was defined as $<20,000$ platelets $/ \mathrm{mm}^{3}$.

Our institutional ethics committee approved this study (no. 01-61-39). All procedures performed in studies involving human participants met the ethical standards set out by institutional and national research guidelines and were in accordance with the 1964 Helsinki declaration and its later amendments. For this retrospective study, formal informed consent was not required.

\section{Results}

Six patients were included in the study; of whom four (67\%) were female. Baseline characteristics, underlying diseases, clinical presentations and clinical outcomes are shown in Tables 1 and 2. None of the patients with hematologic malignancies had a history of hyperglycemia or acidosis. Four patients had relapsed hematologic malignancies (duration: 2, 2, 10, and 11 years) and had previously received multiple blood transfusions. In the two patients in which they were measured, ferritin levels were $2419 \mathrm{ng} / \mathrm{mL}$ and $5074 \mathrm{ng} / \mathrm{mL}$. Among patients with hematologic malignancies $(\mathrm{n}=5), 80 \%$ were neutropenic for a duration of 6-26 days prior to diagnosis. Only one patient had irreversible neutropenia for a duration of 56 days after diagnosis due to myelodysplasia. The sites of infection included sinus-orbit-cerebral (67\%), lung $(17 \%)$, and disseminated infection $(17 \%)$ in a patient with pneumonia. All patients with hematologic malignancies were treated with preemptive antifungals with a median duration of 9 days (range: 3-31 days) prior to receiving a proven diagnosis of IM. Effective antifungals were given within 3-5 days after the onset of symptoms; the reasons for this delay were varied, including infections that occurred on an outpatient basis $(n=2)$, those coincident with bacteremia $(\mathrm{n}=2)$, those coincident with Aspergillus spp. infection $(\mathrm{n}=1)$, and undiagnosed infection before surgery $(n=1)$. Details of antifungal treatment history are described in Table 2. All patients received $\mathrm{AmB}$ as the first-line regimen. DAmB was initially given for patients without renal dysfunction due to financial reasons. The dose of D-AmB ranged from 1$1.5 \mathrm{mg} / \mathrm{kg} /$ day and that of L-AmB ranged from $5-10 \mathrm{mg} / \mathrm{kg} /$ day. Only one patient with rhinocerebral mucormycosis and carotid arteritis received high-dose L-AmB (10 $\mathrm{mg} / \mathrm{kg} /$ day) for 41 days in combination with DEF. Step-down posaconazole was given on an outpatient basis to four patients. One diabetic patient failed oral posaconazole therapy despite adequate therapeutic plasma levels of $4.26 \mathrm{ng} / \mathrm{mL}$. The Mucorales species causing the infection was identified in four patients and included Rhizopus spp.

Table 1. Baseline characteristics of patients with invasive mucormycosis $(n=6)$.

\begin{tabular}{lc} 
Parameter & \\
Age (years; median/range) & $50(19-64)$ \\
Gender (female; $n, \%)$ & $4(67)$ \\
\hline Weight (kg; median/range) & $55(45-65)$ \\
Underlying disease & $5(83)$ \\
Hematologic disease (n, \%) & $1(17)$ \\
Diabetes mellitus (n, \%) & $21(23-7)$ \\
Disease onset post chemotherapy (n=5), days (median/range) & \\
\hline Treatment (n, \%) & $5(83)$ \\
Surgery (n, \%) & \\
Duration of antifungal treatment (days, median/range) & $64(38-114)$ \\
Amphotericin B based regimen (n=6, 100\%) & $26(7-43)$ \\
Echinocandins (n=6, 100\%) & $53(23-353)$ \\
Posaconazole (n=5, 83\%) & $86(46-435)$ \\
Total duration of antifungals (days) & $14(21-56)$ \\
Duration of antifungal therapy prior to deferiprone (days) & \\
\hline Deferiprone (median, range) & $100(86.2-100)$ \\
Doses (mg/kg/day) & $34(10-215)$ \\
Duration of deferiprone treatment (days) & \\
Outcome & $4(67)$ \\
Clinical success at 12 weeks (n, \%) & $5(83)$ \\
Death (n, \%) & $2(33)$ \\
Death due to mucormycosis (n, \%) & $1(17)$ \\
Mortality rate at 72 days (n, \%) & $2(33)$ \\
Mortality rate at 90 days (n, \%) & $317(56-1217)$ \\
Duration of survival after diagnosis (days; median/range) & \\
\hline
\end{tabular}




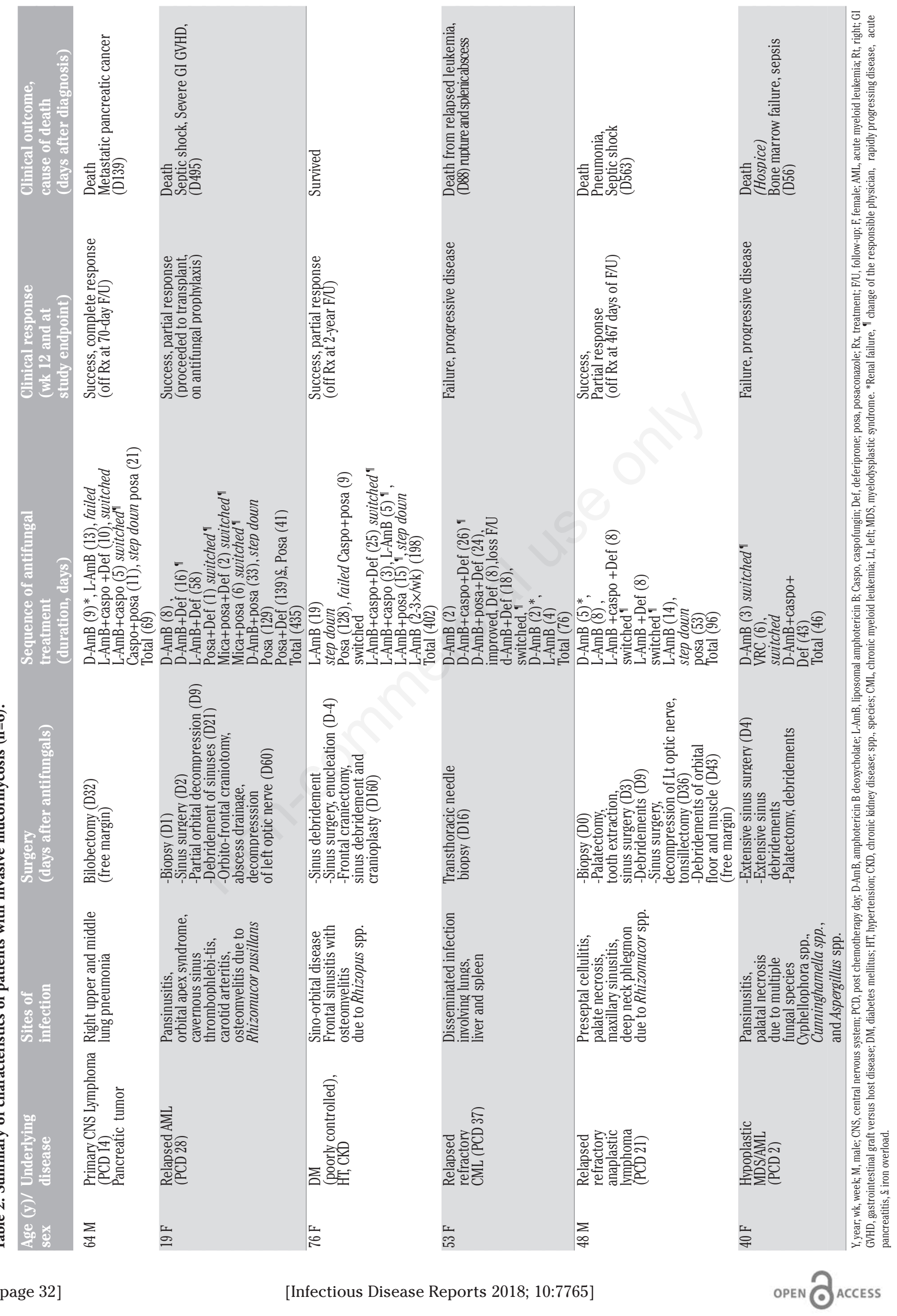


$(\mathrm{n}=1), \quad$ Rhizomucor spp. $\quad(\mathrm{n}=2)$, and Cunninghamella spp. $(\mathrm{n}=1)$. In vitro susceptibility data was not available for the isolates. Most patients underwent surgery $(83 \%)$, the details of which are shown in Table 2. The time elapsed from initiation of antifungal treatment to surgery ranged from 2-31 days. Local control was achieved in $33 \%$ of patients with confined pulmonary disease or sinus infection after radical surgery. All patients with sinus infection underwent repeated radical debridements which caused significant morbidity, including blindness due to enucleation and optic neuritis $(\mathrm{n}=2)$ and disfigurement due to palatectomy and extensive surgery $(n=2)$. Three patients had residual localized disease after surgery (cavernous sinus thrombosis with carotid arteritis, cranial osteomyelitis and dural involvement, pansinusitis and deep neck phlegmon). Antifungal treatments received before initiation of DEF included monotherapy using AmB-based regimens (67\%), voriconazole (17\%), and caspofungin combined with posaconazole $(17 \%)$. The details of these treatments are described in Table 2. DEF was given as adjunctive first-line therapy of IM in five patients due to rapidly progressing disease, despite treatment with a combination of antifungals and/or surgery, and as salvage therapy in one patient. None of the patients received iron chelators at the time of diagnosis. The dose of DEF was modified to suit an alternate day regimen for improved tolerability in two patients after 7 and 27 days of daily treatment, respectively. Treatment with DEF was continued for more than 30 days for refractory IM and iron overload. Ferritin levels in two patients decreased after treatment with DEF. Laboratory data are shown in Table 3 . Potential adverse events defined using CTCAE criteria are shown in Table 4. Grade 2 liver parameter abnormalities (excluding ALT) occurred in two patients (33\%), which led to discontinuation of DEF. One patient had comorbid pancreatic cancer and gallstone pancreatitis. One patient developed acute pancreatitis (mild) relating to high-dose L-AmB (10 mg/kg/day) which led to temporary discontinuation of L-AmB and DEF. DEF was reinitiated one month later due to iron overload, with a reduced dose on an alternate day regimen to reduce nausea. The patient continued to receive medication for 139 days without any further adverse events. Grade 3 elevated serum creatinine levels were observed in three patients $(50 \%)$ and were attributed to DAmB toxicity and tumor lysis syndrome. None of the patients developed rashes. The reasons for drug discontinuation were surgical cure of disease $(n=2)$, medical cure, gas- trointestinal intolerance (nausea and liver dysfunction), and death due to IM $(n=2)$. DEF was discontinued after radical debridement in four patients who showed clinical

improvements. The overall success rate evaluated at week 12 of follow-up was $67 \%$. Two patients achieved a successful clinical outcome by surgical resection of

Table 3. Medians and ranges of laboratory parameters at time of initiation of antifungals and at time of initiation and discontinuation of deferiprone $(n=6)$.

\begin{tabular}{lccc} 
Parameter & Antifungals & Initiation & Discontinuation \\
White blood cell count (cells/mm³) & $4,000(100-93,580)$ & $5,845(100-8030)$ & $8,605(1,100-28,210)$ \\
Absolute neutrophil count & $870(100-72,057)$ & $4347(100-6,989)$ & $6128(517-25,953)$ \\
\hline Hematocrit (\%) & $27.7(25.4-36)$ & $28.1(26-33)$ & $29.1(22-33.7)$ \\
Platelet count (cells/mm³) & $56,000(18,000-214,000)$ & $51,500(17,000-161,000)$ & $79,000(18,000-341,000)$ \\
\hline Albumin (g/L) & $26.6(20-41.5)$ & $21.6(13.8-26.6)$ & $23(15.4-28.7)$ \\
Total bilirubin (mg/dL) & $1.2(0.3-6.9)$ & $1.4(0.5-1.8)$ & $0.8(0.5-1.5)$ \\
\hline Aspartate aminotransferase (U/L) & $29(7-119)$ & $31(11-50)$ & $63(20-99)$ \\
Alanine aminotransferase (U/L) & $32(19-122)$ & $35(11-48)$ & $39(37-82)$ \\
\hline Alkaline phosphatase (U/L) & $89(51-359)$ & $156(51-301)$ & $279(106-348)$ \\
Gamma glutamyltransferase (U/L) & $61(31-521)$ & $121(58-354)$ & $339(54-558)$ \\
\hline Creatinine (mg/dL) & $0.83(0.4-1.55)$ & $1.2(0.48-1.97)$ & $1.3(0.5-1.7)$ \\
\hline
\end{tabular}

Table 4. Assessment of potential adverse effects during deferiprone therapy.

\begin{tabular}{|c|c|c|}
\hline Parameter & Criterion & Deferiprone ( $\mathrm{n}=6), \mathrm{n} / \mathrm{n}(\%)$ \\
\hline White blood cell count (cells/mm ${ }^{3}$ ) & $<1000$ & $1 / 6(16.7)$ \\
\hline Platelet count (cells $\left./ \mathrm{mm}^{3}\right)$ & $\begin{array}{l}<20,000 \\
\text { CTCAE }\end{array}$ & 2/6 (33.3) \\
\hline Anemia & $\begin{array}{l}\text { Grade } 1 \\
\text { Grade } 2 \\
\text { Grade } 3 \\
\text { Grade } 4\end{array}$ & $\begin{array}{c}1 / 6(16.7) \\
4 / 6(66.7) \\
0 \\
0\end{array}$ \\
\hline AST & $\begin{array}{l}\text { Grade } 1 \\
\text { Grade } 2 \\
\text { Grade } 3 \\
\text { Grade } 4\end{array}$ & $\begin{array}{c}0 \\
2 / 6(33.3) \\
0 \\
0\end{array}$ \\
\hline ALT & $\begin{array}{l}\text { Grade } 1 \\
\text { Grade } 2 \\
\text { Grade } 3 \\
\text { Grade } 4\end{array}$ & $\begin{array}{l}0 \\
0 \\
0 \\
0\end{array}$ \\
\hline ALP & $\begin{array}{l}\text { Grade } 1 \\
\text { Grade } 2 \\
\text { Grade } 3 \\
\text { Grade } 4\end{array}$ & $\begin{array}{c}1 / 6(16.7) \\
2 / 6(33.3) \\
0 \\
0\end{array}$ \\
\hline GGT & $\begin{array}{l}\text { Grade } 1 \\
\text { Grade } 2 \\
\text { Grade } 3 \\
\text { Grade } 4\end{array}$ & $\begin{array}{c}0 \\
2 / 6(33.3) \\
1 / 6(16.7) \\
0\end{array}$ \\
\hline Total bilirubin & $\begin{array}{l}\text { Grade } 1 \\
\text { Grade } 2 \\
\text { Grade } 3 \\
\text { Grade } 4\end{array}$ & $\begin{array}{c}0 \\
2 / 6(33.3) \\
0 \\
0\end{array}$ \\
\hline Albumin & $\begin{array}{l}\text { Grade } 1 \\
\text { Grade } 2 \\
\text { Grade } 3 \\
\text { Grade } 4\end{array}$ & $\begin{array}{c}0 \\
4 / 6(66.7) \\
2 / 6(33.3) \\
0\end{array}$ \\
\hline Creatinine & $\begin{array}{l}\text { Grade } 1 \\
\text { Grade } 2 \\
\text { Grade } 3 \\
\text { Grade } 4\end{array}$ & $\begin{array}{c}0 \\
0 \\
3 / 6(50) \\
0\end{array}$ \\
\hline
\end{tabular}

CTCAE, Common Terminology Criteria for Adverse Events (version 5.0); AST, aspartate transaminase; ALT, alanine transaminase; ALP, alkaline phosphatase; GGT, gamma glutamyltransferase. 
localized disease combined with medical therapy. Three patients showed no evidence of relapse after discontinuation of antifungals, and one patients underwent stem cell transplantation and received antifungal prophylaxis without documented infection. The median duration of survival after diagnosis was 317 days (range: 56-1,217) days. The mortality rate at 42-, 72-, and 180-day follow-up was $0 \%, 17 \%$, and $50 \%$, respectively. Death due to IM occurred in two patients within 56 and 88 days of diagnosis, respectively. Causes of death are shown in Table 2.

\section{Discussion}

Our study showed preliminary safety and tolerability data of adjunctive DEF for treatment of mucormycosis in conjunction with antifungals and surgery. DEF was initiated as adjunctive treatment for IM based on its preclinical efficacy against Mucorales $^{7}$ and treatment of presumed transfusion-induced iron overload among patients with relapse hematologic malignancies. Ferritin levels were not measured in all patients given that its measurement and interpretation may be difficult during active inflammation. DEF was also administered to a diabetic patient who failed to respond to combined antifungals and surgery based on the clinical efficacy of deferasirox in diabetic patients. ${ }^{11}$ Success outcomes at 12-week follow-up, as determined by EORTC/MSG criteria, were achieved in four patients. ${ }^{22}$ Death occurred in two patients with unfavorable risk factors including relapsed hematologic malignancy, cytopenia, hypoalbuminemia, and infection with Cunninghamella spp. which is more resistance to antifungals..$^{13,23-25}$ The mortality rate in our study was lower than previous studies for multiple potential reasons. ${ }^{12-13,26,27}$ Firstly, most patients with sinus disease underwent repeated wide surgical debridements to achieve local control, which is associated with survival benefit. ${ }^{28}$ Secondly, most patients had localized IM and reversible neutropenia, both of which are associated with better outcomes. ${ }^{2,29}$ Thirdly, preemptive therapy with $\mathrm{AmB}$ was given to patients with hematologic malignancies within 5 days of admission, which associated with lower mortality rate. ${ }^{2}$ Fourthly, combination therapy with AmB plus echinocandin was administered, which has been associated with improved outcomes in rhinocerebral mucormycosis. ${ }^{30}$ However, the conflicting data from recent analyses did not achieve statistical significance. ${ }^{29}$ Treatment-emergent adverse events were reported in $50 \%$ of the patients. The most common adverse events were nausea, vomiting, and hepatobiliary tract disorders. Drug-related adverse events were reported in $33 \%$ of patients and were reversible with drug discontinuation. Our study had several limitations; as a single-center retrospective case series examining a small number of patients due to rarity of the disease, recruiting a control group to conduct a case-control study was not feasible. A previous multicenter randomized control trial (DEFEAT trial) involved only 20 patients, 11 of whom were randomized to the iron chelator arm. ${ }^{12}$ Our study did not include transplant recipient, immunosuppressed or critical care patient. Another potential limitation was the limited number of sites of infection and infecting species of Mucorales observed here, and the generalizability of our results to other settings is unknown. Several other confounding factors, including antifungals regimen and timing and impact of surgery, may have contributed to the success outcomes in our study. Future case-control or prospective studies as multicenter collaborations to reach larger sample sizes should be conducted in order determine the efficacy of iron chelators in IM. ${ }^{18}$

\section{Conclusions}

Mucormycosis is a life-threatening disease with rapid progression among immunosuppressed patients. Early diagnosis and prompt treatment with Mucoralesactive antifungals and surgery to achieve local control remain the primary treatment for the disease. In real-life experience, adjunctive treatment with DEF showed safety and tolerability.

\section{References}

1. Cornely OA, Arikan-Akdagli S, Dannaoui E, et al. ESCMID and ECMM joint clinical guidelines for the diagnosis and management of mucormycosis 2013. Clin Microbiol Infect 2014;20:5-26.

2. Chamilos G, Lewis RE, Kontoyiannis DP. Delaying amphotericin B-based frontline therapy significantly increases mortality among patients with hematologic malignancy who have zygomycosis. Clin Infect Dis 2008;47:503-9.

3. Kontoyiannis DP, Yang H, Song J, et al. Prevalence, clinical and economic burden of mucormycosis-related hospitalizations in the United States: a retrospective study. BMC Infect Dis 2016;16:730.

4. Tissot F, Agrawal S, Pagano L, et al.
ECIL-6 guidelines for the treatment of invasive candidiasis, aspergillosis and mucormycosis in leukemia and hematopoietic stem cell transplant patients. Haematologica 2017;102:43344.

5. Barton JC, Bertoli LF. Transfusion iron overload in adults with acute leukemia: manifestations and therapy. Am J Med Sci 2000;319:73-8.

6. Ibrahim AS. Host-iron assimilation: pathogenesis and novel therapies of mucormycosis. Mycoses 2014;57:13-7.

7. Ibrahim AS, Edwards JE, Jr., Fu Y, et al. Deferiprone iron chelation as a novel therapy for experimental mucormycosis. J Antimicrob Chemother 2006;58: 1070-3.

8. Ibrahim AS, Gebermariam T, Fu Y, et al. The iron chelator deferasirox protects mice from mucormycosis through iron starvation. J Clin Invest 2007; 117:264957.

9. Lewis RE, Pongas GN, Albert N, et al. Activity of deferasirox in Mucorales: influences of species and exogenous iron. Antimicrob Agents Chemother 2011;55:411-3.

10. Reed C, Ibrahim A, Edwards JE, et al. Deferasirox, an iron-chelating agent, as salvage therapy for rhinocerebral mucormycosis. Antimicrob Agents Chemother 2006;50:3968-9.

11. Spellberg B, Andes D, Perez M, et al. Safety and outcomes of open-label deferasirox iron chelation therapy for mucormycosis. Antimicrob Agents Chemother 2009;53:3122-5.

12. Spellberg B, Ibrahim AS, Chin-Hong $\mathrm{PV}$, et al. The deferasirox-ambisome therapy for mucormycosis (DEFEAT Mucor) study: a randomized, doubleblinded, placebo-controlled trial. J Antimicrob Chemother 2012;67:71522.

13. Roden MM, Zaoutis TE, Buchanan WL, et al. Epidemiology and outcome of zygomycosis: a review of 929 reported cases. Clin Infect Dis 2005;41:634-53.

14. Spellberg B, Ibrahim AS. Recent advances in the treatment of mucormycosis. Curr Infect Dis Rep 2010;12:4239.

15. Shoham S, Magill SS, Merz WG, et al. Primary treatment of zygomycosis with liposomal amphotericin B: analysis of 28 cases. Med Mycol 2010;48:511-7.

16. Llorente A, Perez-Valero I, Garcia E, et al. Mortality risk factors in patients with zygomycosis: a retrospective and multicentre study of 25 cases. Enferm Infecc Microbiol Clin 2011;29:263-8.

17. Soman R, Gupta N, Shetty A, et al. Deferasirox in mucormycosis: hopeful- 
ly, not defeated. J Antimicrob Chemother 2012;67:783-4.

18. Donnelly JP, Lahav M. Deferasirox as adjunctive therapy for mucormycosis. $\mathrm{J}$ Antimicrob Chemother 2012;67:51920.

19. Taher A, Sheikh-Taha M, Sharara A, et al. Safety and effectiveness of 100 $\mathrm{mg} / \mathrm{kg}$ /day deferiprone in patients with thalassemia major: a two-year study. Acta Haematol 2005;114:146-9.

20. Belmont A, Kwiatkowski JL. Deferiprone for the treatment of transfusional iron overload in thalassemia. Exp Rev Hematol 2017;10:493-503.

21. De Pauw B, Walsh TJ, Donnelly JP, et al. Revised definitions of invasive fungal disease from the European Organization for Research and Treatment of Cancer/Invasive Fungal Infections Cooperative Group and the National Institute of Allergy and Infectious Diseases Mycoses Study Group (EORTC/MSG) Consensus Group. Clin Infect Dis 2008;46:1813-
21.

22. Segal BH, Herbrecht R, Stevens DA, et al. Defining responses to therapy and study outcomes in clinical trials of invasive fungal diseases: Mycoses Study Group and European Organization for Research and Treatment of Cancer Consensus Criteria. Clin Infect Dis 2008;47:674-83.

23. Gomes MZ, Lewis RE, Kontoyiannis DP. Mucormycosis caused by unusual mucormycetes, non-Rhizopus, -Mucor, and -Lichtheimia species. Clin Microbiol Rev 2011;24:411-45.

24. Davoudi S, Kumar VA, Jiang Y, et al. Invasive mould sinusitis in patients with haematological malignancies: a 10 year single-centre study. J Antimicrob Chemother 2015;70:2899-905.

25. Pagano L, Akova M, Dimopoulos G, et al. Risk assessment and prognostic factors for mould-related diseases in immunocompromised patients. J Antimicrob Chemother 2011;66:i5-i14. 26. Kennedy KJ, Daveson K, Slavin MA, et al. Mucormycosis in Australia: contemporary epidemiology and outcomes. Clin Microbiol Infect 2016;22:775-81.

27. Marty FM, Ostrosky-Zeichner L, Cornely OA, et al. Isavuconazole treatment for mucormycosis: a single-arm open-label trial and case-control analysis. Lancet Infect Dis 2016;16:828-37.

28. Vironneau P, Kania R, Morizot G, et al. Local control of rhino-orbito-cerebral mucormycosis dramatically impacts survival. Clin Microbiol Infect 2014;20:336-9.

29. Kyvernitakis A, Torres H, Jiang Y, et al. Initial use of combination treatment does not impact early survival of 106 patients with hematologic malignancies and mucormycosis: a propensity score analysis. Mycoses 2016;22:811.e1-8.

30. Reed C, Bryant R, Ibrahim AS, et al. Combination polyene-caspofungin treatment of rhino-orbital-cerebral mucormycosis. Clin Infect Dis 2008;47:364-71. 